\title{
A Rare Case of Falciform Ligament Abscess with Unknown Etiology
}

\author{
${ }^{1}$ Vimal K Jain, ${ }^{2}$ Ashishkumar G Hadiyal, ${ }^{3}$ Shalit A Jolly, ${ }^{4}$ Vivek Maurya
}

\begin{abstract}
The incidence of a falciform ligament is very rare. Because of the rarity of the condition and sparsity of available literature, it's very difficult to diagnose this condition preoperatively. In this case, a 65-year-old lady had presented with pain in epigastrium and vomitings for 3 days. All blood investigations were normal except serum Alkaline phosphatase (ALP) and serum gamma-glutamyl transferase (GGT) which were $141 \mathrm{IU} / \mathrm{L}$ and $275 \mathrm{U} / \mathrm{L}$ respectively. USG revealed only chronic cholecystitis. On diagnostic laparoscopy, falciform ligament abscess was detected which was adequately drained. The patient responded well with the drainage without recurrence till date. Laparoscopic cholecystectomy was also done in the same sitting.
\end{abstract}

Keywords: Cholecystitis, Diagnostic laparoscopy, Falciform ligament abscess.

List of Used Abbreviations: ALP-Alkaline phosphatase, CECT-Contrast enhanced computed tomography, DNB-Diplomates of national board, ECG-Electrocardiography, GGTGamma-glutamyl transferase, PAC-Pre-anesthetic clearance, RBS-Random blood sugar, SGOT-Serum glutamic oxaloacetic transaminase, SGPT-Serum glutamic-pyruvic transaminase, USG-Ultrasonography

How to cite this article: Jain VK, Hadiyal AG, Jolly SA, Maurya V. A Rare Case of Falciform Ligament Abscess with unknown Etiology. World J Lap Surg 2018;11(2):103-105.

Source of support: Nil

Conflict of interest: None

\section{INTRODUCTION}

The falciform ligament helps in holding the liver in place by attaching it to the anterior abdominal wall. It is composed of two layers of peritoneum closely united together which might provide potential space for infection. Its free edge contains the round ligament (ligamentum teres) and the paraumbilical veins between its layers. This sickle-shaped ligament (Latin-falciform means sickle) is remnant of the fetal umbilical vein and derivative of the embryonic ventral mesentery.

\footnotetext{
${ }^{1}$ Senior Consultant Surgeon, ${ }^{3}$ Junior Consultant Surgeon ${ }^{2,4}$ DNB Trainee

${ }^{1-4}$ Department of General Surgery, Mata Chanan Devi Hospital, Janakpuri, New Delhi, India
}

Corresponding Author: Ashishkumar G Hadiyal, DNB Trainee, Department of General Surgery, Mata Chanan Devi Hospital, Janakpuri, New Delhi, India, e-mail: ashish_hadiyal3@yahoo.com
The incidence of the falciform ligament is very rare. Only a few cases have been reported till date. The pathophysiology of abscess formation in falciform ligament is not very well understood. Preoperatively diagnosis of this condition is difficult. A case of falciform ligament abscess misdiagnosed as acute cholecystitis in our hospital is reported here.

\section{Clinical Case Presentation}

A female patient aged 65 years admitted in Mata Chanan Devi hospital with a complaint of pain in epigastrium and vomitings for 2 to 3 days.

The pain was severe, sudden in onset, constant, radiating to back and not related to the meal-no previous history of such type of pain. There was no history of fever, jaundice, breathing difficulty or any trauma to chest and abdomen. There was no history of surgery in the past. The patient was nonalcoholic and not a diabetic or hypertensive and had no other major illnesses.

The patient was afebrile, pulse rate was $82 /$ minute and blood pressure was 118/74 $\mathrm{mm}$ of $\mathrm{Hg}$. The abdomen was tender in the epigastrium and right hypochondrium. There was no palpable mass. As per history and clinical examination, a provisional diagnosis of acute cholecystitis was made.

Complete blood count, RBS, serum electrolytes, urea, creatinine, amylase, lipase, bilirubin, SGOT, SGPT, chest X-ray, ECG were normal. Serum ALP was mildly raised(141 IU/L) GGT was raised (275 U/L). USG findings were suggestive of chronic cholecystitis with cholelithiasis.

Provision diagnosis of acute cholecystitis was made while USG findings were not in favor. After PAC patient underwent diagnostic laparoscopy. On operation, the falciform ligament was bulging and was firm in consistency (Fig. 1). On aspiration, the purulent material came out (Fig. 2). All purulent material was drained by giving a cruciate incision and wound was irrigated adequately with normal saline (Fig. 3). Laparoscopic cholecystectomy was done. HPE confirmed the diagnosis of chronic cholecystitis with cholelithiasis. The patient responded well. She was discharged on after 2 days. The patient was followed up for 60 days. There were no symptoms suggestive of recurrence. 


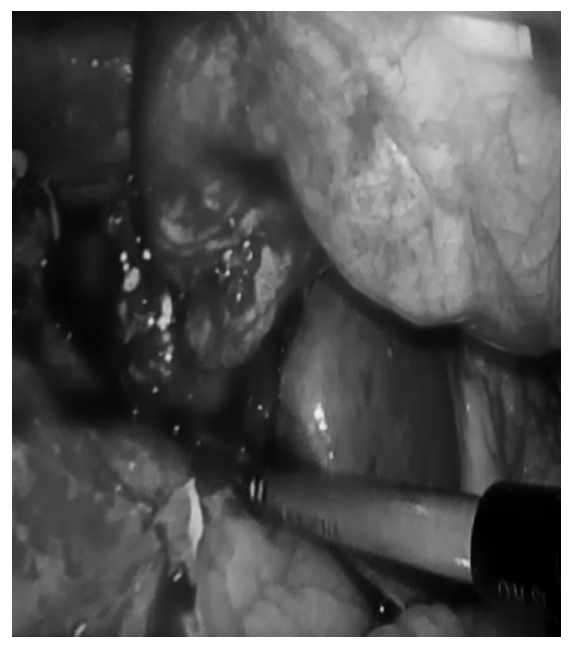

Fig. 1: Intraoperative image showing bulging falciform ligament

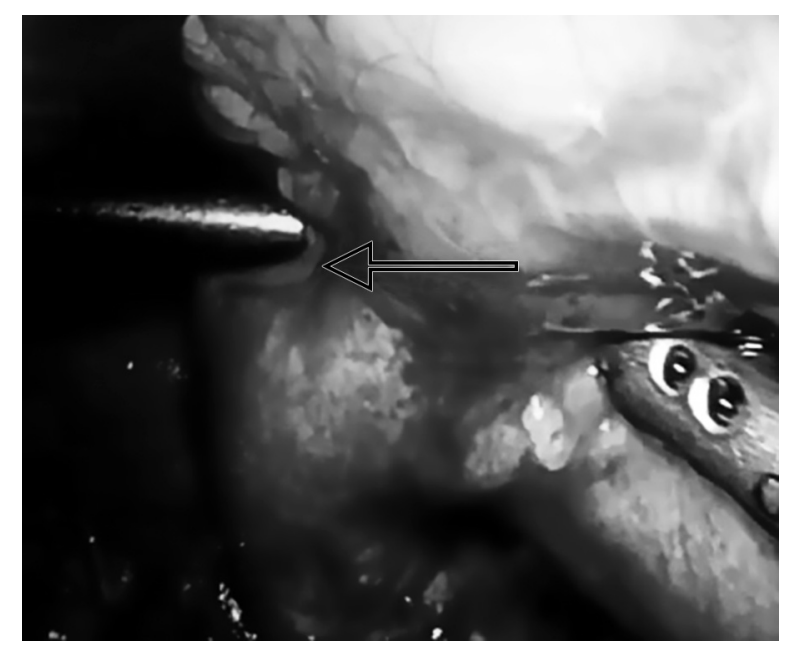

Fig. 2: Intraoperative image showing purulent material on aspiration

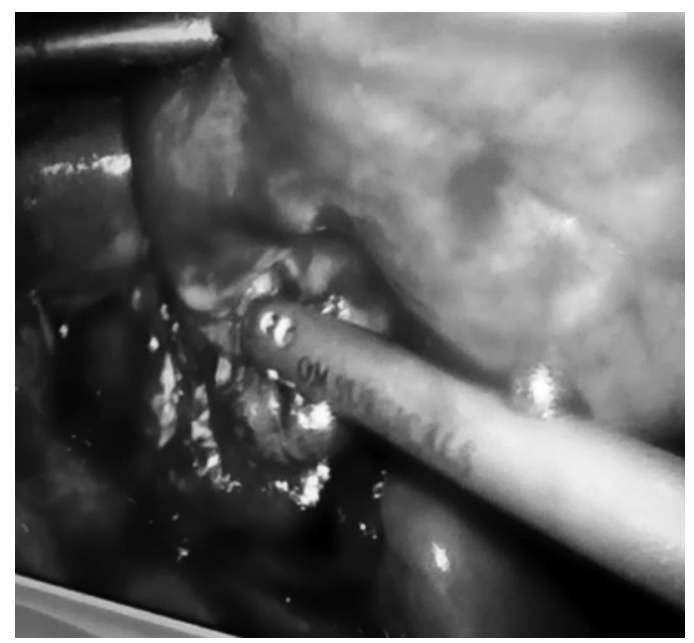

Fig. 3: Intraoperative image showing cruciate shaped incision was given

\section{DISCUSSION}

Surgical lesions of the falciform ligament are rare. Clinically, they present most often as a cystic abdominal mass and less often as an abscess. ${ }^{1}$ Very few cases of this condition have been reported worldwide. One such case was reported in the patient suspected of acute cholecystitis clinically, but computed tomography revealed a cylindrical mass in the anterior abdomen with the possibility of a hepatic abscess. On laparoscopy, falciform ligament abscess was found. It was drained at the same time. After two months there was a recurrence of the abscess of falciform ligament secondary to acute calculous cholecystitis. ${ }^{2}$ Other few cases of this condition have been reported in the setting of rupture of the gangrenous gallbladder, ${ }^{3}$ portal pyemias, ${ }^{4,5}$ post omphalitis, ${ }^{6,7}$ post pancreatitis, ${ }^{8}$ and infected ventriculoperitoneal shunt. ${ }^{9}$ There is one case of an isolated falciform ligament necrosis presenting as acute abdomen has also been reported. ${ }^{10,11}$

Accurate preoperative diagnosis of falciform ligament abscess could not be made in cases as reported in the literature. Since exact etiopathology is still poorly understood, a strong index of suspicion is needed for early diagnosis and management of this condition. It has been observed that infection can extend from liver, gallbladder or umbilicus to the falciform ligament. ${ }^{2-6}$ Though in this case it was found that gallbladder was not acutely inflamed and no infective lesion was present in the abdominal cavity. Here, relationship with chronic cholecystitis could not be established. In previously reported cases treatment with percutaneous drainage and antibiotics was tried but resulted in recurrence. ${ }^{2-6}$ This might be explained by the scarcity of the vascular network inside the ligamentous structure that hampers exposure to the circulation of antibiotics. ${ }^{6}$ So percutaneous drainage is not a preferred treatment option for this condition.

\section{CONCLUSION}

A high index of suspicion is needed to diagnose this condition. Typically it presents with pain in the epigastrium or right hypochondrium, with or without fever, leucocytosis and palpable abdominal mass. USG is not 
much useful. CECT may help in reaching to the diagnosis. Diagnostic laparoscopy is an investigation of choice. It is diagnostic as well as therapeutic for this condition. Though percutaneous drainage of falciform ligament abscess was tried in few cases, patients had a recurrence in all the cases. But laparoscopic abscess drainage and debridement of the falciform ligament with post operative antibiotic coverage are sufficient to treat this condition.

\section{REFERENCES}

1. Brock JS, Pachter HL, Schreiber J, Hofstetter SR. Surgical diseases of the falciform ligament. American Journal of Gastroenterology. 1992 Jun 1;87(6):757-758.

2. Melo VA, Melo GB, Silva RL, Aragão JF, Rosa JE. Falciform ligament abscess: report of a case. Revista do Hospital das Clínicas. 2003;58(1):37-38.

3. Sones Jr PJ, Thomas BM, Masand PP. Falciform ligament abscess: appearance on computed tomography and sonography. American Journal of Roentgenology. 1981 Jul 1;137(1):161-162.

4. Warren LR, Chandrasegaram MD, Madigan DJ, Dolan PM, Neo EL, Worthley CS. Falciform ligament abscess from left sided portal pyaemia following malignant obstructive cholangitis. World journal of surgical oncology. 2012 Dec;10(1):278.

5. Moon SB, Lee HW, Park KW, Jung SE. Falciform ligament abscess after omphalitis: report of a case. Journal of Korean medical science. 2010 Jul 1;25(7):1090-1020.

6. Suk-Bae Moon et al. Falciform ligament abscess after omphalitis: report of a case.J Korean Med Sci 2010 Jul 16;25(7):10901092. Epub 2010 Jun 16.

7. Atif QA, Khaliq T. Postpancreatitis abscess of falciform ligament: an unusual presentation. J Coll Physicians Surg Pak. 2015 Nov 1;25:837-838.

8. Laucks SS, Ballantine TV, Boal DK. Abscess of the falciform ligament in a child with a ventriculoperitoneal shunt. Journal of pediatric surgery. 1986 Nov 1;21(11):979-980.

9. Ozkececı ZT, Ozsoy M, Celep B, Bal A, Polat C. A rare cause of acute abdomen: an isolated falciform ligament necrosis. Case reports in emergency medicine. 2014.

10. Lipinski JK, Vega JM, Cywes S, Cremin BJ. Falciform ligament abscess in the infant. Journal of pediatric surgery. 1985 Oct 1;20(5):556-558.

11. Losanoff JE, Kjossev KT. Isolated gangrene of the round and falciform liver ligaments: a rare cause of peritonitis: case report and review of the world literature. The American Surgeon. 2002 Sep 1;68(9):751. 